\title{
The Diamond Lemma and the PBW Property in Quantum Algebras
}

\author{
M. Havlíček, S. Pošta
}

\begin{abstract}
The use of the diamond lemma for proving various facts about the center of the algebra $U_{q}^{\prime}\left(\mathrm{so}_{3}\right)$ is demonstrated. The approach presented here is successful in other cases of quantum algebras and superalgebras.
\end{abstract}

Keywords: quantum algebra, PBW property, center.

\section{Introduction}

A fundamental issue when examining quantum groups or similar structures is to explore and classify all representations.

Usually one encounters two main cases which are of different difficulty. When the deformation parameter, in quantum groups typically denoted by $q$, is not a root of unity, i. e. when $q^{n} \neq 1$ for all integers $n$, the representation theory is "the same" as in the classical case, that is in the case of enveloping algebras of classical Lie algebras. When $q$ is a root of unity, it is not an easy task to classify finite dimensional representations even in the lowest dimensions.

Various preparatory steps must be taken before one can investigate representations of the considered algebra. Important supporting knowledge which helps in making such a classification is detailed information about the center of the considered algebra, because irreducible representation operators which belong to the center of the algebra (Casimir operators) are represented by a scalar multiple of the unit operator.

The Harish-Chandra homomorphism (1951) is an isomorphism which maps the center $Z(U(g))$ of the universal enveloping algebra $U(g)$ of a semisimple Lie algebra $g$ to the elements $S(h)^{W}$ of the symmetric algebra $S(h)$ of a Cartan subalgebra $h \subset g$ that are invariant under the corresponding Weyl group $W$.

Let $r$ be the rank of $g$, which is the dimension of the Cartan subalgebra $h$. Coxeter observed that $S(h)^{W}$ is a polynomial algebra in $r$ variables. Therefore, the center of the universal enveloping algebra of a semisimple Lie algebra is a free polynomial ring. Any Casimir operator is an arbitrary polynomial in basic algebra invariants. The number and degrees of these fundamental invariants are shown in Table 1.

In the case of standard Drinfeld-Jimbo quantum groups, we have an analogy to the non-deformed case. When deformation parameter $q$ is not a root of unity, a modified version of the Harish-Chandra homomorphism exists and the center is again isomorphic to the ring of polynomials of the fundamental invariants (see [1]).
Table 1: Degrees of the fundamental invariants

\begin{tabular}{lc}
\hline$A_{r}$ & $2,3,4, \ldots, n+1$ \\
$B_{r}$ & $2,4,6, \ldots, 2 n$ \\
$C_{r}$ & $2,4,6, \ldots, 2 n$ \\
$D_{r}$ & $n ; 2,4,6, \ldots, 2 n-2$ \\
$E_{6}$ & $2,5,6,8,9,12$ \\
$E_{7}$ & $2,6,8,10,12,14,18$ \\
$E_{8}$ & $2,8,12,14,18,20,24,30$ \\
$F_{4}$ & $2,6,8,12$ \\
$G_{2}$ & 2,6 \\
\hline
\end{tabular}

For example, in the simplest case of the algebra $U_{q}\left(\mathrm{sl}_{2}\right)$, generated by four letters (generators) denoted by $E, F, K, K^{-1}$ and relations

$$
\begin{aligned}
K K^{-1} & =K^{-1} K=1, \\
K E K^{-1} & =q^{2} E \\
K F K^{-1} & =q^{-2} F \\
{[E, F] } & \equiv E F-F E=[K]_{q} \equiv \frac{K-K^{-1}}{q-q^{-1}},
\end{aligned}
$$

the center is generated by the Casimir element

$$
C_{q}=E F+\frac{K q^{-1}+K^{-1} q}{\left(q-q^{-1}\right)^{2}}
$$

(for the standard proof of this fact see [2], theorem 45).

When $q$ is a primitive root of unity, say $q^{n}=1$, $n>1, q^{m} \neq 1$ for $m<n$, the situation is much more difficult. The center is typically much larger and the central elements satisfy nontrivial polynomial relations [3]. In the case of $U_{q}\left(\mathrm{sl}_{2}\right)$, there are four more additional elements in the center, namely

$$
E^{p}, \quad F^{p}, \quad K^{p}, \quad K^{-p},
$$

where $p=n$ if $n$ is odd and $p=\frac{n}{2}$ if $n$ is even. These five elements (together with (1)) are no longer algebraically independent. One can show by induction 
that

$\prod_{j=0}^{p-1}\left(C_{q}-\left(q-q^{-1}\right)^{-2}\left(K q^{j+1}+K^{-1} q^{-j-1}\right)\right)=E^{p} F^{p}$,

which implies

$$
\begin{aligned}
& C_{q}^{p}+\gamma_{1} C_{q}^{p-1}+\ldots+\gamma_{p-1} C_{q}+ \\
& (-1)^{p}\left(q-q^{-1}\right)^{-2 p}\left(K^{p}-K^{-p}\right)=E^{p} F^{p},
\end{aligned}
$$

where $\gamma_{i}$ are certain complex coefficients.

Quantum groups are not the only kind of quantum deformations. There exist also other, nonstandard deformations. For example, $q$-deformation $U_{q}^{\prime}\left(\mathrm{SO}_{3}\right)$ of the universal enveloping algebra $U\left(\mathrm{sO}_{3}\right)$, which does not coincide with the Drinfeld-Jimbo quantum algebra $U_{q}\left(\mathrm{SO}_{3}\right)$ is constructed without using the Cartan subalgebra and roots by deforming Serre-type relations directly. We substitute simply $2 \rightarrow[2]_{q}=q+q^{-1}$ in cubic defining relations of $U\left(\mathrm{SO}_{3}\right)$. As a result we obtain a complex associative algebra with unity generated by elements $I_{21}, I_{32}$ satisfying the relations

$$
\begin{aligned}
& I_{21}^{2} I_{32}-\left(q+q^{-1}\right) I_{21} I_{32} I_{21}+I_{32} I_{21}^{2}=-I_{32}, \\
& I_{21} I_{32}^{2}-\left(q+q^{-1}\right) I_{32} I_{21} I_{32}+I_{32}^{2} I_{21}=-I_{21} .
\end{aligned}
$$

It can be shown that this is isomorphic to an algebra generated by three generators $I_{1}, I_{2}, I_{3}$ and relations $[5]$

$$
\begin{aligned}
& q^{\frac{1}{2}} I_{1} I_{2}-q^{-\frac{1}{2}} I_{2} I_{1}=I_{3}, \\
& q^{\frac{1}{2}} I_{2} I_{3}-q^{-\frac{1}{2}} I_{3} I_{2}=I_{1} \\
& q^{\frac{1}{2}} I_{3} I_{1}-q^{-\frac{1}{2}} I_{1} I_{3}=I_{2}
\end{aligned}
$$

One can quickly explore the following Casimir element, which belongs to the center of this algebra:

$$
C=q^{2} I_{1}^{2}+I_{2}^{2}+q^{2} I_{3}^{2}-\left(q^{\frac{5}{2}}-q^{\frac{1}{2}}\right) I_{1} I_{2} I_{3} .
$$

Similarly as in the case of ordinary Hopf quantum groups, one can expect that when $q$ is not a root of unity, this element generates the center of the algebra $U_{q}^{\prime}\left(\mathrm{so}_{3}\right)$. However, there is no analogy of HarishChandra homomorphism here so one must prove this fact by other methods. As is shown below, these methods are useful even in the more complicated case when $q$ is a root of unity.

\section{The diamond lemma}

In 1978, M. Bergmann recalled a rather deep and forgotten result of Newman from graph theory, often called the diamond lemma. He showed its usefulness for other fields of mathematics also, namely for the theory of associative algebras. The original Newman formulation was as follows (see [6]). Let $G$ be an oriented graph. Now suppose that
1) The oriented graph $G$ has the descending chain condition. That is, all positively oriented paths in $G$ terminate, in other words, there are no circles in the graph.

2) Whenever two edges, $e$ and $e^{\prime}$, proceed from one vertex $a$ of $G$, there exist positively oriented paths $p, p^{\prime}$ in $G$ leading from the endpoints $b, b^{\prime}$ of these edges to a common vertex $c$. (This is often called the "confluence" or "diamond" condition, see fig. 1.)

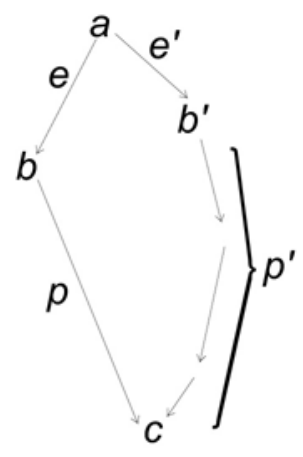

Fig. 1: Diamond condition

Then one can show that every connected component $C$ of $G$ has a unique minimal vertex $m_{C}$. This means that every maximal positively oriented path beginning at a point of $C$ will terminate at $m_{C}$.

Let us now describe the version of the diamond lemma in the theory of associative algebras. Let $R$ be an associative algebra with unity over complex numbers, given by the finite set of generators $X$ and the set of relations

$$
S=\left\{W_{\sigma}=f_{\sigma} \mid \sigma \in \Sigma\right\}
$$

where $W_{\sigma}$ is monomial (the product of a finite number of generators from $X$ ) and $f_{\sigma}$ is a complex linear combination of monomials. Let us have partial ordering $<$ defined on monomials which satisfies three conditions: it is invariant with respect to multiplication, i. e. for each monomial $A, B, B^{\prime}, C$ we have

$$
B<B^{\prime} \Longrightarrow A B C<A B^{\prime} C
$$

it is $S$-compatible (i. e. $f_{\sigma}$ is a linear combination of elements being less than $W_{\sigma}$ for each $\sigma \in \Sigma$ ) and fulfils DCC (the descending chain condition, which is the nonexistence of an infinite sequence $\left.x_{1}>x_{2}>\ldots\right)$. Furthermore, let all monomials which can be written as product $A B C$, where $A B=W_{\sigma}$ and $B C=W_{\tau}, B \neq 1, \sigma, \tau \in \Sigma$ or in the form $A B C=W_{\sigma}$ and $B=W_{\tau}, \sigma \neq \tau$ (these monomials are called ambiguities) be reduced by the relations in $S$ to a common value. Then the diamond lemma states that all irreducible (i. e. completely reduced, to which one cannot apply any relation from $S$ ) monomials form a basis of algebra $R$. 
The diamond lemma can be effectively used for deciding various kinds of problems. The typical problem, as mentioned in [6], is as follows. Let us have an algebra with generators $a, b, c$ and the relations

$$
\begin{gathered}
a^{2}=a, \quad b^{2}=b, \quad c^{2}=c \\
(a+b+c)^{2}=a+b+c
\end{gathered}
$$

Now the problem is to answer the question whether it follows from these relations that $a b=0$. The second relation (5) can be rewritten as

$$
c b=-a b-b a-a c-c a-b c .
$$

Now we test whether (4), (5) and (6) imply a unique canonical form of the elements of the considered algebra. We must examine the following ambiguities:

$$
a^{3}, \quad b^{3}, \quad c^{3}, \quad c b^{2}, \quad c^{2} b .
$$

The first three are trivial to reduce, and the fourth, reduced in two possible ways, gives us the following:

$$
c(b b)=c b=-a b-b a-a c-c a-b c
$$

and

$$
\begin{aligned}
(c b) b= & (-a b-b a-a c-c a-b c) b= \\
& -a b^{2}-b a b-a c b-c a b-b c b= \\
& -a b-b a b-a(-a b-b a-a c-c a-b c)- \\
& c a b-b(-a b-b a-a c-c a-b c)= \\
& -a b-b a b+a^{2} b+a b a+a^{2} c+ \\
& a c a+a b c-c a b+b a b+ \\
& b^{2} a+b a c+b c a+b^{2} c= \\
& -a b-b a b+a b+a b a+a c+a c a+ \\
& a b c-c a b+b a b+b a+b a c+b c a+b c= \\
& a b a+a c+a c a+a b c-c a b+ \\
& b a+b a c+b c a+b c .
\end{aligned}
$$

So we have

$$
\begin{aligned}
& -a b-b a-a c-c a-b c= \\
& a b a+a c+a c a+a b c-c a b+b a+b a c+b c a+b c .
\end{aligned}
$$

This equality can be rewritten as

$$
\begin{aligned}
c a b= & a b a+2 a c+a c a+a b c+ \\
& 2 b a+b a c+b c a+2 b c+a b+c a .
\end{aligned}
$$

Reducing the fifth ambiguity

$$
c(c b)=(c c) b
$$

leads to the same relation. Now what happens if we add (7) to the list of relations? The ambiguities $c b^{2}$ and $c^{2} b$ now reduce automatically to a common value. But two new ambiguities of higher degree arise:

$$
c^{2} a b, \quad c a b^{2} \text {. }
$$

We therefore test again: we have

$$
\begin{aligned}
(c c) a b=c a b= & a b a+2 a c+a c a+a b c+2 b a+ \\
& b a c+b c a+2 b c+a b+c a,
\end{aligned}
$$

and, after some computation,

$$
\begin{aligned}
c(c a b)=\ldots= & a b a+2 a c+a c a+a b c+2 b a+ \\
& b a c+b c a+2 b c+a b+c a .
\end{aligned}
$$

The second ambiguity reduces to a common value, too. The basis of the considered algebra consists of all words consisting of letters $a, b, c$ not containing substrings $a^{2}, b^{2}, c^{2}, c a b$ and $c b$. Therefore the word $a b$ is irreducible, hence nonzero.

\section{PBW property}

One of simple consequences of the diamond lemma is the Poincaré-Birkhoff-Witt property of universal enveloping algebras and its analogy in the case of quantum deformations.

As a simple example, let us have an enveloping algebra $U\left(\mathrm{sl}_{2}\right)$ of Lie algebra $\mathrm{sl}_{2}$ which is given by three generators $E, F, H$ satisfying the relations

$$
\begin{aligned}
& {[E, F]=E F-F E=H,} \\
& {[H, E]=2 E,} \\
& {[H, F]=-2 F .}
\end{aligned}
$$

We define the total ordering $\prec$ of the generators as it is in the alphabet, i. e. $E \prec F \prec H$. Partial ordering among monomials is defined in such way that $X<Y$, when the length of $X$ (the number of letters in the product $X$ ) is less than the length of $Y$, or when $X$ is a permutation of the letters from $Y$, but has a lower number of inverses (the monomial $X=x_{1} \ldots x_{s}$ has inverse $(i, j), 1 \leq i, j \leq s$, when $i<j$ and $\left.x_{i} \succ x_{j}\right)$. One can easily see that this partial ordering is compatible with relations (8), which we present in a more suitable form of "rewriting rules":

$$
\begin{aligned}
& F E \rightarrow E F-H, \\
& H E \rightarrow E H+2 E, \\
& H F \rightarrow F H-2 F .
\end{aligned}
$$

One can also easily check that the ordering fulfils DCC. Simple computation gives us

$$
\begin{aligned}
(H F) E= & (F H-2 F) E=F H E-2 F E= \\
& F(E H+2 E)-2 F E= \\
& F E H=(E F-H) H=E F H-H^{2}, \\
H(F E)= & H(E F-H)=H E F-H^{2}= \\
& (E H+2 E) F-H^{2}= \\
& E H F+2 E F-H^{2}= \\
& E(F H-2 F)+2 E F-H^{2}= \\
& E F H-2 E F+2 E F-H^{2}= \\
& E F H-H^{2} .
\end{aligned}
$$


We see that the ambiguity HFE is reduced to a common value. One can easily list all irreducible monomials. These are precisely

$$
E^{\alpha} F^{\beta} H^{\gamma}, \quad \alpha, \beta, \gamma \geq 0 .
$$

The diamond lemma states that these monomials form the basis of the algebra $U\left(\mathrm{sl}_{2}\right)$ (as stated by the well known PBW theorem).

\section{Center of $U\left(\mathrm{sl}_{2}\right)$}

As was said in the introduction, there is a standard way to explore the structure of the center of the algebra $U\left(\mathrm{sl}_{2}\right)$. Let us find this structure now using the diammond lemma. This procedure can then be generalized to other algebras where standard tools like the Harish-Chandra homomorphism cannot be used.

The problem is to find all elements $X$ from $U\left(\mathrm{sl}_{2}\right)$, for which we have

$$
[X, A] \equiv X A-A X=0 \quad \text { for all } A \in U\left(\mathrm{sl}_{2}\right) \text {. }
$$

This condition is clearly equivalent to

$$
[X, E]=[X, F]=[X, H]=0,
$$

that is, one can restrict oneself to making commutations with generators only. Let us take a general element of the form

$$
X=\sum_{i, j, k=0}^{n} \alpha_{i, j, k} E^{i} F^{j} H^{k}
$$

for some small values of $n$, let us generally compute commutators $[X, E],[X, F]$ a $[X, H]$ and solve a system of linear equations (10) for coefficients $\alpha_{i, j, k}$ only).

For $n=1$ we get nothing (trivial zero solution

For $n=2$ we get any scalar multiple of

$$
X=H^{2}+4 E F-2 H \equiv C .
$$

For higher $n$ we get elements of the form

$$
\alpha C+\beta C^{2}
$$

then

$$
\alpha C+\beta C^{2}+\gamma C^{3}
$$

etc., where $\alpha, \beta, \gamma, \ldots$ are arbitrary complex coefficients.

Of course this leads to the hypothesis that any element $X$ which commutes with $E, F$ and $H$ is of the form $p(C)$, where $p$ is an arbitrary complex polynomial.

The proof is based on the change of the original basis $\left\{E^{\alpha} F^{\beta} H^{\gamma}\right\}$. We add to generators $E, F$ and
$H$ another letter $C$ and to the rewriting rules (9) we add the following:

$$
\begin{aligned}
& E F \rightarrow \frac{1}{4}\left(C+2 H-H^{2}\right), \\
& E C \rightarrow C E, \\
& F C \rightarrow C F, \\
& H C \rightarrow C H .
\end{aligned}
$$

The basis now consists of irreducible elements, i. e.

$$
\left\{C^{j} E^{k} H^{m} \mid j, k, m \geq 0\right\} \cup\left\{C^{j} F^{l} H^{m} \mid j, l, m \geq 0\right\} .
$$

We now take the general element as a linear combination

$X=\sum_{j, k, m=0}^{n} \beta_{j, k, m} C^{j} E^{k} H^{m}+\sum_{j, l, m=0}^{n} \gamma_{j, l, m} C^{j} F^{l} H^{m}$.

When computing commutators $[X, E],[X, F]$ and $[X, H]$ one can make use of the fact that, for example

$$
\left[C^{j} E^{k} H^{m}, E\right]=C^{j}\left[E^{k} H^{m}, E\right]=C^{j} E^{k}\left[H^{m}, E\right]
$$

etc. It is also clear (as opposed to the original case) what it is sufficient to show: one must show that coefficients $\beta_{j, k, m}=0$ for $(k, m) \neq(0,0)$, similarly for $\gamma$ 's. This can be seen from (10).

\section{Center of $U_{q}^{\prime}\left(\mathrm{so}_{3}\right)$}

Let us apply the process introduced above to the case of the nonstandard deformation $U_{q}^{\prime}\left(\mathrm{so}_{3}\right)$. First, let us assume $q^{n} \neq 1$ for all $n$.

Using the first relation (2) as a definition for $I_{3}$ and substituting into the second and third relations we get two cubic relations

$$
\begin{aligned}
& I_{2} I_{1}^{2}-\left(q+q^{-1}\right) I_{1} I_{2} I_{1}+I_{1}^{2} I_{2}=-I_{2}, \\
& I_{2}^{2} I_{1}-\left(q+q^{-1}\right) I_{2} I_{1} I_{2}+I_{1} I_{2}^{2}=-I_{1} .
\end{aligned}
$$

It can be shown that $U_{q}^{\prime}\left(\mathrm{so}_{3}\right)$ is isomorphic to the algebra with the generators $I_{1}, I_{2}$ satisfying the two above relations. The casimir element (3) can be rewritten to the form (we ommit scalar factor $q$ )

$C=\left(q+q^{-1}\right)\left(I_{1}^{2}+I_{1}^{2} I_{2}^{2}+I_{2}^{2}\right)+I_{2} I_{1} I_{2} I_{1}-[3]_{q} I_{1} I_{2} I_{1} I_{2}$.

We now construct a different basis of the algebra $U_{q}^{\prime}\left(\mathrm{so}_{3}\right)$. We deal with the following rewriting rules:

$$
\begin{aligned}
I_{2} I_{1}^{2} \rightarrow & \left(q+q^{-1}\right) I_{1} I_{2} I_{1}-I_{1}^{2} I_{2}-I_{2}, \\
I_{2}^{2} I_{1} \rightarrow & \left(q+q^{-1}\right) I_{2} I_{1} I_{2}-I_{1} I_{2}^{2}-I_{1}, \\
I_{2} I_{1} I_{2} I_{1} \rightarrow & C+[3]_{q} I_{1} I_{2} I_{1} I_{2}- \\
& \left(q+q^{-1}\right)\left(I_{1}^{2}+I_{1}^{2} I_{2}^{2}+I_{2}^{2}\right), \\
I_{2} C \rightarrow & C I_{2}, \\
I_{1} C \rightarrow & C I_{1} .
\end{aligned}
$$


It can easily be seen that precisely the elements

$$
C^{\gamma} I_{1}^{\alpha}\left(I_{2} I_{1}\right)^{k} I_{2}^{\beta}, \quad \gamma, \alpha, \beta \geq 0, k \in\{0,1\}
$$

are irreducible, thus forming a new linear basis of the algebra.

Now it is sufficient to take arbitrary element $X$ of the form

$$
X=\sum_{\alpha, \beta, k} p_{\alpha, \beta, k}(C) I_{1}^{\alpha}\left(I_{2} I_{1}\right)^{k} I_{2}^{\beta}
$$

where $p_{\alpha, \beta, k}$ are arbitrary complex polynomials of one variable, commute $X$ with $I_{1}, I_{2}$ and by virtue of its equality to zero to show that all polynomials $p_{\alpha, \beta, k}=0$ with the only exception $p_{0,0,0}$.

When $q$ is a primitive root of unity, $q^{n}=1$, the center of the algebra does not only consist of the ordinary Casimir element (3) but there are three more elements having the form

$$
C_{n 1}=\sum_{j=0}^{\left[\frac{n-1}{2}\right]}\left(\begin{array}{c}
n-j \\
j
\end{array}\right) \frac{n}{n-j}\left(\frac{i}{q-q^{-1}}\right)^{2 j} I_{1}^{n-2 j}
$$

(and the same polynomial in $I_{2}$ and $I_{3}$ denoted by $\left.C_{n 2}, C_{n 3}\right)$. It is not an easy task to show that these elements really belong to the center of the algebra (see [4]). After some transformation one can see that $C_{n j}, j=1,2,3$ are actually Chebyshev polynomials.

It turns out that these four Casimir elements are no longer polynomial independent. When one wants to prove this fact it may come in handy to have explicit polynomial dependence for small values of $n$. However, it turns out that it is practically impossible to obtain the relation between Casimir elements by "brute force", even for the simplest cases. For example, for $n=3$ one can show that

$$
\begin{gathered}
C^{3}-q C^{2}-C_{31}^{2}-C_{32}^{2}- \\
C_{33}^{2}+3\left(q+q^{\frac{1}{2}}\right) C_{31} C_{32} C_{33}=0 .
\end{gathered}
$$

Note that $C^{3}$ is of degree nine, so it is quite complicated even to prove the given explicit relation.

The diamond lemma can be quite useful for obtaining relations of type (11) directly. We must only construct a suitable set of rewriting rules and with the help of it new advantageous basis of the algebra.

First we start with ordinary rewriting rules coming from the commutation relations, namely

$$
\begin{aligned}
& I_{2} I_{1} \rightarrow q I_{1} I_{2}-q^{\frac{1}{2}} I_{3}, \\
& I_{3} I_{2} \rightarrow q I_{2} I_{3}-q^{\frac{1}{2}} I_{1}, \\
& I_{3} I_{1} \rightarrow q^{-1} I_{1} I_{3}+q^{-\frac{1}{2}} I_{2}, \\
& I_{1} C \rightarrow C I_{1}, \\
& I_{2} C \rightarrow C I_{2}, \\
& I_{3} C \rightarrow C I_{3} .
\end{aligned}
$$

The powers of the generators can be reduced using Casimir elements $C_{n k}$, therefore we add the relations

$$
I_{k}^{n} \rightarrow C_{n k}-\sum_{j=1}^{\left[\frac{n-1}{2}\right]}\left(\begin{array}{c}
n-j \\
j
\end{array}\right) \frac{n}{n-j}\left(\frac{i}{q-q^{-1}}\right)^{2 j} I_{k}^{n-2 j},
$$

$k=1,2,3$.

Next we must ensure that Casimir element $C$ does not appear too many times. This is ensured by the relation

$$
A C B \rightarrow A\left(q^{2} I_{1}^{2}+I_{2}^{2}+q^{2} I_{3}^{2}-\left(q^{\frac{5}{2}}-q^{\frac{1}{2}}\right) I_{1} I_{2} I_{3}\right) B
$$

where $A, B$ are arbitrary monomials, and it comes into play only if the count of letters $I_{k}(k=1,2,3)$ and $C$ in monomial $A C B$ is greater or equal to $n$. The last rule (or, better to say, set of rules) which we do not present explicitly transforms

$$
C^{j} W \rightarrow C^{j+1} \tilde{W}
$$

where $W$ is any product of generators $I_{k}, k=1,2,3$ with the property that every generator $I_{1}, I_{2}, I_{3}$ is present in the product. Using the commutation relations, $W$ is transformed to the form $I_{1} I_{2} I_{3} W_{1}$ and the product $I_{1} I_{2} I_{3}$ is then converted to $C$ using the rule

$$
I_{1} I_{2} I_{3} \rightarrow\left(q^{\frac{5}{2}}-q^{\frac{1}{2}}\right)^{-1}\left(q^{2} I_{1}^{2}+I_{2}^{2}+q^{2} I_{3}^{2}-C\right) .
$$

These transformation rules now lead to the basis containing the following elements:

$$
\begin{aligned}
& C_{n 1}^{\alpha} C_{n 2}^{\beta} C_{n 3}^{\gamma} C^{\delta} I_{1}^{k} I_{2}^{l} I_{3}^{m}, \\
& \alpha, \beta, \gamma \geq 0,0 \leq \delta \leq n-1, \\
& 0 \leq k, l, m \leq n-1-\delta, \quad k l m=0 .
\end{aligned}
$$

If we want to find the explicit relation between Casimir elements, we simply express $C^{n}$ in the basis specified (using a finite number of rewriting rules reducing $C^{n}$ to canonical form).

In general, one can show that for odd $n$ the elements $C, C_{n 1}, C_{n 2}, C_{n 3}$ fulfil the relation

$$
\begin{aligned}
& C_{n 1}^{2}+C_{n 2}^{2}+C_{n 3}^{2}+\left(q-q^{-1}\right)^{n} C_{n 1} C_{n 2} C_{n 3}= \\
& \prod_{k=0}^{n-1}\left(C+q[k]_{q}[k+1]_{q}\right) .
\end{aligned}
$$

The relation for even $n$ as well as the proof of this can be found in [7].

\section{Acknowledgement}

We acknowledge financial support from grant 201/10/1509 of the Czech Science Foundation and MSM6840770039 of the Ministry of Education, Youth, and Sports of the Czech Republic. 


\section{References}

[1] Lusztig, G: Introduction to Quantum Groups, Birkhäuser, Boston, 1993.

[2] Klimyk, A. U., Schmüdgen, K.: Quantum groups and their representations, Springer, Berlin, 1997.

[3] Tange, R.: The centre of quantum $s l_{n}$ at root of unity, J. Algebra 301 (2006) 425-445.

[4] Havlíček, M., Pošta, S.: On the classification of irreducible finite-dimensional representations of $U_{q}^{\prime}\left(\mathrm{SO}_{3}\right)$ algebra, J. Math. Phys. 42 (2001), 472-500.

[5] Havlíček, M., Klimyk, A. U., Pošta, S.: Representations of the cyclically symmetric $q$-deformed algebra so $(3)$, J. Math. Phys. 40 (1999), 2 135-2 161.

[6] Bergmann, G. M.: Diamond Lemma for ring theory, Adv. Math. 29 (1978), 178-218.

[7] Havlíček, M., Pošta, S.: Center of algebra $U_{q}^{\prime}\left(\mathrm{so}_{3}\right)$, submitted to J. Math. Phys.

Prof. Ing. Miloslav Havlíček, DrSc.

E-mail: miloslav.havlicek@fjfi.cvut.cz doc. Ing. Severin Pošta, Ph.D.

E-mail: severin.posta@fjfi.cvut.cz Department of Mathematics

FNSPE, Czech Technical University in Prague

Trojanova 13, CZ-120 00 Prague 